\title{
Effect of Variable Viscosity on Hydro Magnetic Flow and Heat Transfer Over a Stretching Surface with Variable Temperature
}

\author{
M. Y. Akl \\ Department of Basic Science, Faculty of Engineering (Shopra Branch), \\ Banha University, Cairo, Egypt.
}

A mathematical model is presented to study the role of an applied magnetic field on heat transfer in a fluid flow over a stretching surface in the presence of temperature dependent viscosity. A similarity transformation is used to reduce the governing partial differential equations into ordinary ones, which are solved numerically by shooting method. Numerical results for the velocity and temperature profiles as well as for the skin friction and Nusselt number are obtained and reported graphically for various parametric conditions to show interesting aspects of the solution.

\section{Introduction:}

Recently, the study of nonlinear hydro magnetic flow and heat transfer over a stretching surface has received considerable interest, because of its wide applicability in energy, such as geothermal energy technology, petroleum recovery, glass fiber production, metal extrusion, hot rolling, the cooling and/or drying of paper and textiles, and wire drawing.

Most of the existing analytical studies for this problem are based on the constant physical properties of the ambient fluid [1-3]. However, it is known that these properties may change with temperature [4]. To accurately predict the flow and heat transfer rates it is necessary to take into account this variation of viscosity. The study of heat transfer and the flow field is necessary for determining the quality of the final products of these processes as explained by Karwe and Jaluria [5].

E-mail: mhmdyhak1@yahoo.com 
In studying the motion of such a fluid, the non-linearity of the basic equation and additional mathematical difficulties associated with it has led several investigators to explore the perturbation and numerical methods. Hydrodynamic flows of a viscous and incompressible fluid have been studied under different physical conditions with variable fluid properties by Hassanien [4] and Seddeek [6-8]. In many particle engineering system, both the plane surface and the ambient fluid are moving in parallel.

The steady flow of an electrically conducting fluid caused solely by the stretching of an elastic sheet in the presence of a uniform magnetic field was investigated by Pavlov [9]. The flow and heat transfer of an electrically conducting incompressible fluid past a porous wall stretching linearly was considered by Chakrabarti and Gupta [10]. Chiam [11] reported solutions for steady hydromagnetic flow over a surface stretching with a power-law velocity with the distance along the surface. Up to author's knowledge, no attempt has been made so far on the study of nonlinear MHD flow and heat transfer over a surface of variable temperature stretching with a power-law velocity.

Hence, the aim of the present work is to study the effects of variable viscosity and magnetic field on hydromagnetic flow and heat transfer over a surface of variable temperature stretching with a power-law velocity.

\section{Problem formulation:}

Consider a two-dimensional steady flow of a laminar, incompressible fluid past a flat surface issuing from a very thin slit at $\mathrm{x}=0, \mathrm{y}=0$ and subsequently being stretched, as in a polymer extrusion process and subjected to a transverse variable magnetic field $\mathrm{B}(\mathrm{x})$ (neglecting viscous and Joule's dissipation). We assume that the fluid properties are isotropic and constant, except for the fluid viscosity $\mu$, which is assumed to vary as an inverse linear function of temperature $\mathrm{T}$, in the form [12].

$$
\begin{array}{llll}
\frac{1}{\mu}=\frac{1}{\mu_{\infty}}\left(1+\delta\left(T-\mathrm{T}_{\infty}\right)\right) & \text { or } & \frac{1}{\mu}=\mathrm{E}\left(\mathrm{T}-\mathrm{T}_{\mathrm{r}}\right) \\
\text { where } \mathrm{E}=\frac{\delta}{\mu_{\infty}} & \text { and } & \mathrm{T}_{\mathrm{r}}=\mathrm{T}_{\infty}-\frac{1}{\delta}
\end{array}
$$

where $\mu_{\infty}$ and $T_{\infty}$ are the fluid free stream dynamic viscosity and the fluid free-stream temperature. $\mathrm{E}$ and $\mathrm{T}_{r}$ are constants and their values depend on the reference state and thermal property of the fluid, i. e. $\delta$. In general, $\mathrm{E}>0$ for fluid such as liquids and $\mathrm{E}<0$ for gases. Let us assume that the speed of a point on the surface is proportional to the power of its distance from the slit and the 


$$
\begin{gathered}
\frac{\partial u}{\partial x}+\frac{\partial v}{\partial y}=0 \\
u \frac{\partial u}{\partial x}+v \frac{\partial u}{\partial y}=\frac{\partial}{\partial y}\left(\frac{\mu}{\rho} \frac{\partial u}{\partial y}\right)-\frac{\sigma B^{2}(x)}{\rho} u \\
u \frac{\partial T}{\partial x}+v \frac{\partial T}{\partial y}=\frac{k}{\rho c_{p}} \nabla^{2} T
\end{gathered}
$$

where $\mathrm{u}$ and $\mathrm{v}$ are the velocity components along the $\mathrm{x}$ - and $\mathrm{y}$ - direction, $\mathrm{k}, \sigma$ and $c_{p}$ are the thermal conductivity, electrical conductivity and the specific heat at constant pressure, respectively. $\rho$ the density of the fluid.

The boundary conditions governing the flow are

at $\mathrm{y}=0: \mathrm{u}=\mathrm{U}(\mathrm{x})=\mathrm{ax}^{\mathrm{m}}, \mathrm{v}=0, \mathrm{~T}=\mathrm{T}_{\mathrm{w}}(\mathrm{x})$

as $\mathrm{y} \rightarrow \infty: \mathrm{u} \rightarrow 0 \quad$ and $\mathrm{T} \rightarrow \mathrm{T}_{\infty}$

where $\mathrm{m}$ is the index of power-law velocity, $\mathrm{T}_{\mathrm{w}}(\mathrm{x})=T_{\infty}+\mathrm{bx}{ }^{\mathrm{n}}$ the wall temperature and $\mathrm{a}$ and $\mathrm{b}$ are constants. $\mathrm{n}$ is the index of power-law variation of wall temperature. The special form for magnetic field $\mathrm{B}(\mathrm{x})=B_{0} x^{(m-1) / 2}$ is chosen to obtain the similarity solution, where $m$ is the index of power-law velocity. Now, we introduce the following dimensionless variables:

$$
\begin{aligned}
& \mathrm{u}=\frac{\partial \psi}{\partial y}=\mathrm{U}(\mathrm{x}) \mathrm{f}^{\prime}(\eta), \mathrm{v}=-\frac{\partial \psi}{\partial x}, \theta(\eta)=\frac{\mathrm{T}-\mathrm{T}_{\infty}}{\mathrm{T}_{\mathrm{w}}-\mathrm{T}_{\infty}} \\
& \theta_{\mathrm{r}}=\frac{\mathrm{T}_{\mathrm{r}}-\mathrm{T}_{\infty}}{\mathrm{T}_{\mathrm{w}}-\mathrm{T}_{\infty}}=-\frac{1}{\delta\left(\mathrm{T}_{\mathrm{w}}-\mathrm{T}_{\infty}\right)} \\
& \eta(\mathrm{x}, \mathrm{y})=\left(\frac{(1+\mathrm{m}) \mathrm{U}(\mathrm{x})}{2 v \mathrm{x}}\right)^{\frac{1}{2}} \mathrm{y}, \psi(\mathrm{x}, \mathrm{y})=\left(\frac{2 v \mathrm{x} \mathrm{U}(\mathrm{x})}{(1+\mathrm{m})}\right)^{\frac{1}{2}} \mathrm{f}(\eta)
\end{aligned}
$$

Substituting expression (5) into the Eqs. $(1-4)$, we get

$$
\mathrm{f}^{\prime \prime \prime}-\frac{1}{\theta-\theta_{\mathrm{r}}} \mathrm{f}^{\prime \prime} \theta^{\prime}-\frac{\theta-\theta_{\mathrm{r}}}{\mathrm{N} \theta_{\mathrm{r}}}\left(\mathrm{ff}^{\prime \prime}-\beta \mathrm{f}^{\prime 2}-\mathrm{Mf} \mathrm{f}^{\prime}\right)=0
$$


$\theta^{\prime \prime}+\operatorname{Pr}\left(\mathrm{f} \theta^{\prime}-\frac{2 \mathrm{n}}{m+1} \mathrm{f}^{\prime} \theta\right)=0$

The transformed boundary conditions are

at $\eta=0: \mathrm{f}=0, \mathrm{f}^{\prime}=1, \theta=1$

as $\eta \rightarrow \infty: \quad f^{\prime}=0, \theta=0$

where primes denotes partial differentiation with respect to the variable $\eta$ and the dimensionless parameters are defined as

$\operatorname{Pr}=\frac{\mu \mathrm{c}_{\mathrm{p}}}{\mathrm{k}}$ (Prandtl number), $\mathrm{M}=\frac{\sigma \mathrm{B}_{\circ}^{2}}{\rho \mathrm{a} \mathrm{m}}$ (Magnetic field parameter), $\mathrm{N}=\frac{\mu_{\infty}}{\rho \gamma \mathrm{m}}$ (constant) and $\beta=\frac{\mathrm{m}+1}{2}$ (stretching parameter).

The important physical quantities of our interest are the skin friction $f^{\prime \prime}(0)$ and Nusselt number $-\theta^{\prime}(0)$, and these values tabulated in Table (1). In the case of $\theta_{\mathrm{r}} \rightarrow \infty$, Eqns. (6 and 7) are reduced to that of Anjali and Thiyagarajan [13].

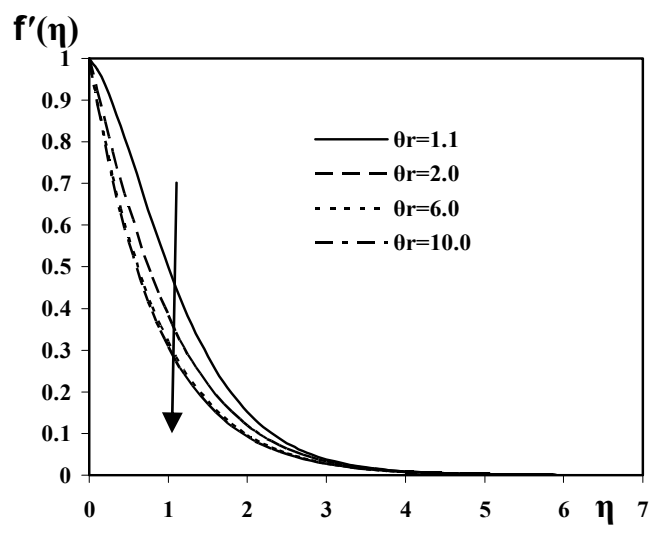

Fig. (1): Velocity distribution for various values of $\theta_{\mathrm{r}}$ at $\mathrm{N}=1, \mathrm{n}=1, \mathrm{~m}=1$, $\operatorname{Pr}=0.72, \mathrm{M}=0.5$ and $\beta=1$

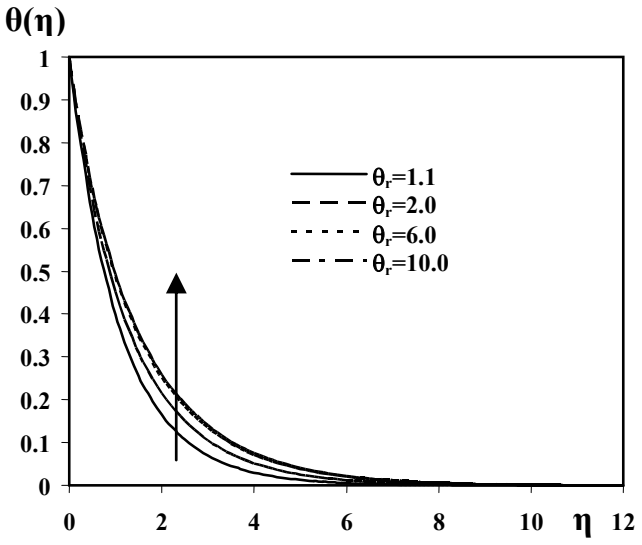

Fig. (2): Temperature distribution for various values of $\theta_{r}$ at $N=1, n=1$, $\operatorname{Pr}=0.72, \mathrm{M}=0.5$ and $\beta=1$ 

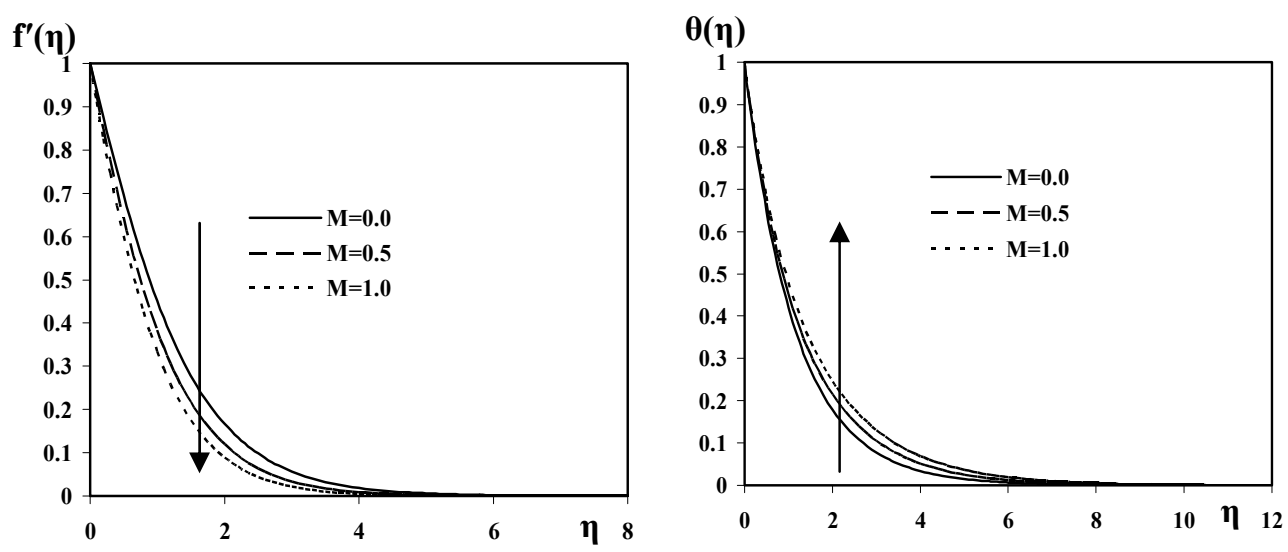

Fig. (3): Velocity distribution for various values of $\mathrm{M}$ at $\mathrm{N}=1, \mathrm{n}=1, \mathrm{~m}=1$, $\operatorname{Pr}=0.72, \theta_{\mathrm{r}}=2$, and $\beta=1$

Fig. (4): Temperature distribution for various values of $\mathrm{M}$ at $\mathrm{N}=1$, $\mathrm{n}=1, \mathrm{~m}=1, \operatorname{Pr}=0.72, \theta_{\mathrm{r}}=2$, and $\beta=1$

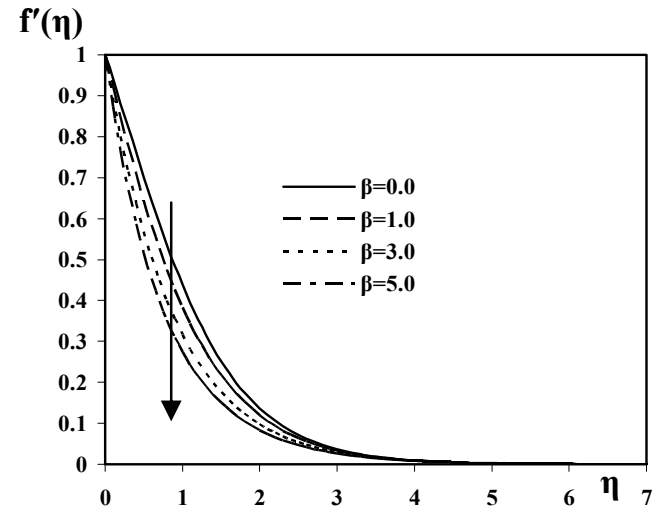

Fig. (5): Velocity distribution for various values of $\beta$ at $\mathrm{N}=1, \mathrm{n}=1, \mathrm{~m}=1$, $\operatorname{Pr}=0.72, \theta_{\mathrm{r}}=2$ and $\mathrm{M}=0.5$

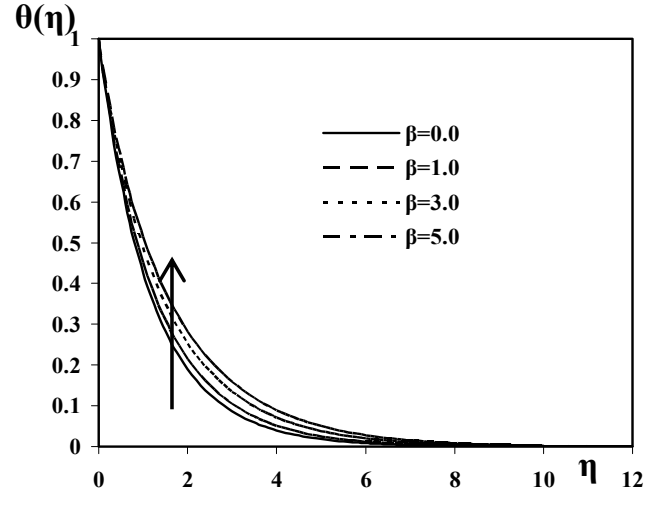

Fig. (6): Temperature distribution for various values of $\beta$ at $N=1, n=1$, $\mathrm{m}=1, \operatorname{Pr}=0.72, \theta_{\mathrm{r}}=2$, and $\mathrm{M}=0.5$ 


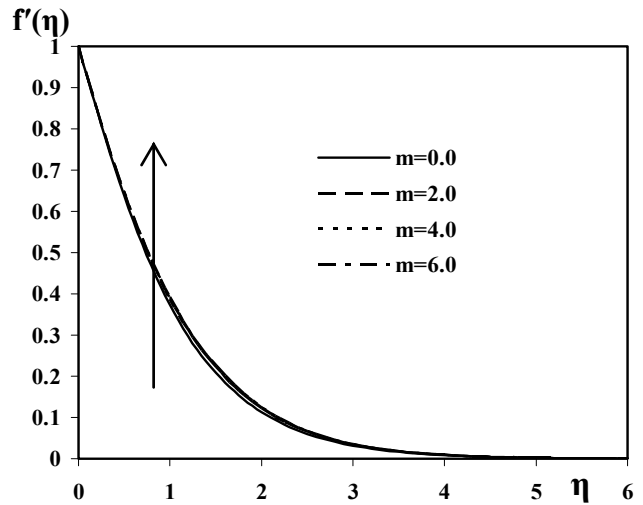

Fig. (7): Velocity tion for various values of $\mathrm{m}$ at $\mathrm{N}=1, \mathrm{n}=1, \mathrm{M}=0.5, \mathrm{Pr}=0.72$, $\theta_{\mathrm{r}}=2$ and $\beta=1$

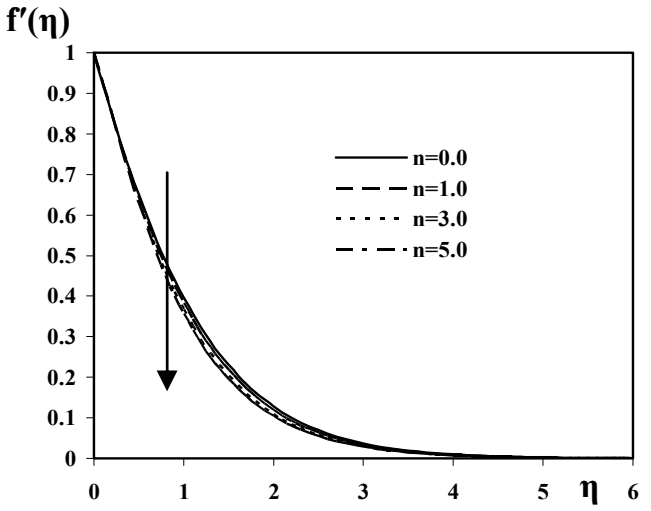

Fig. (9): Velocity tion for various values of $\mathrm{n}$ at $\mathrm{N}=1, \beta=1, \mathrm{~m}=1$, $\operatorname{Pr}=0.72, \theta_{\mathrm{r}}=2$ and $\mathrm{M}=0.5$

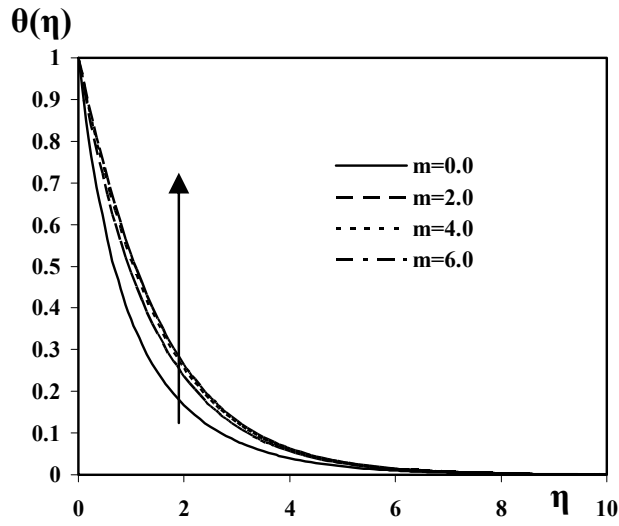

Fig. (8): Temperature distribution for various values of $\mathrm{m}$ at $\mathrm{N}=1, \mathrm{n}=$ 1 ,

$\mathrm{M}=0.5, \operatorname{Pr}=0.72, \theta_{\mathrm{r}}=2$, and $\beta=1$

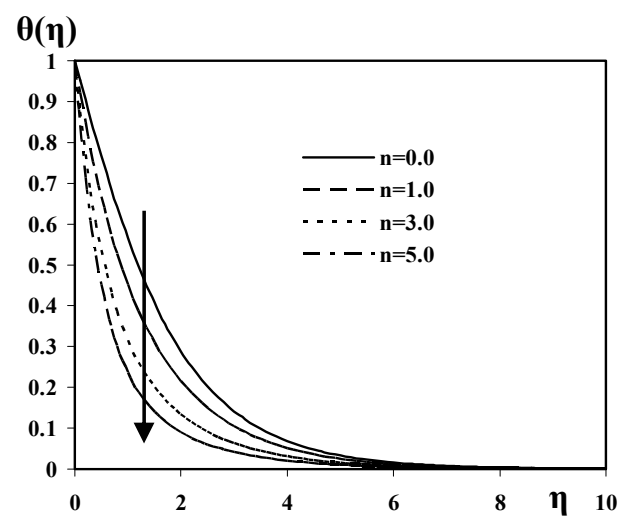

Fig. (10): Temperature distribution for various values of $\mathrm{n}$ at $\mathrm{N}=1, \beta=1$, $\mathrm{m}=1, \operatorname{Pr}=0.72, \theta_{\mathrm{r}}=2$ and $\mathrm{M}=0.5$ 
Table (1): Values of $f^{\prime \prime}(0)$ and $-\theta^{\prime}(0)$ for different values of $\boldsymbol{\theta}_{\mathbf{r}}, \mathrm{M}$ and $\boldsymbol{\beta}$ with $\mathrm{N}=1, \mathrm{n}=1, \mathrm{~m}=1$ and $\mathrm{Pr}=0.72$.

\begin{tabular}{|c|c|c|c|c|}
\hline $\boldsymbol{\theta}_{\mathbf{r}}$ & $\mathbf{M}$ & $\boldsymbol{\beta}$ & $\mathrm{f}^{\prime \prime}(0)$ & $-\theta^{\prime}(0)$ \\
\hline 1.1 & 0.5 & 1.0 & -0.1860 & 0.9051 \\
2 & 0.5 & 1.0 & -0.7769 & 0.8237 \\
6 & 0.5 & 1.0 & -1.0953 & 0.7729 \\
10 & 0.5 & 1.0 & -1.1490 & 0.7639 \\
2 & 0.0 & 1.0 & -0.6284 & 0.8664 \\
2 & 0.5 & 1.0 & -0.7769 & 0.8237 \\
2 & 1.0 & 1.0 & -0.9056 & 0.7879 \\
2 & 0.5 & 0.0 & -0.5744 & 0.8603 \\
2 & 0.5 & 1.0 & -0.7769 & 0.8237 \\
2 & 0.5 & 3.0 & -1.0910 & 0.7710 \\
2 & 0.5 & 5.0 & -1.3420 & 0.7327 \\
\hline
\end{tabular}

\section{Numerical Solution:}

The governing Eqns. (6 \& 7) with the corresponding boundary conditions, Eqn. (8), have been solved numerically by means of the fourth-order Runge-Kutta method with systematic estimates of $f^{\prime \prime}(0)$ and $\theta^{\prime}(0)$ by shooting technique. The step size $\Delta \eta=0.05$ is used while obtaining the numerical solution with $\eta_{\max }=12$ and five-decimal accuracy as the criterion for convergence.

\section{Results and Discussion:}

In this section, a comprehensive numerical parametric study is conducted and the results are reported in terms of graphs. This is done in order to illustrate special features of the solutions.

Figures $(1 \& 2)$ present typical profiles for the velocity and temperature for various values of $\theta_{\mathrm{r}}$, respectively. It is clearly seen that as $\theta_{\mathrm{r}}$ increases the boundary layer thickness decreases and the velocity distribution shallow. Also, the temperature distribution increases, this occurs because for a given fluid, larger $\theta_{\mathrm{r}}$ implies higher temperature difference between the surface and the ambient fluid.

Figures $(3 \& 4)$ illustrate the influence of the magnetic parameter $\mathrm{M}$ on the velocity and temperature profiles in the boundary layer, respectively. Application of a transverse magnetic field to an electrically conducting fluid gives rise to a resistive-type force called the Lorentz force. This force has the 
tendency to slow down the motion of the fluid in the boundary layer and to increase its temperature. Also, the effects on the flow and thermal fields become more so as the strength of the magnetic field increases.

Figures $(5 \& 6)$ give the effects of stretching parameter $\beta$ on the velocity and temperature distributions, respectively. As shown, the velocity distribution decreases with increasing the parameter $\beta$, but the temperature increases with increasing it. We noted that the effect of stretching parameter is to reduce the thickness of the boundary layer.

The effect of the index of power-law velocity $\mathrm{m}$ on the dimensionless velocity and temperature distributions is displayed in Fig. 7 and 8. As shown the dimensionless velocity and the dimensionless temperature increase as the parameter $\mathrm{m}$ increases.

It's seen from Figs. $(9 \& 10)$ that, the variation of distribution of velocity $f^{\prime}(\eta)$ and temperature $\theta(\eta)$ for several values of the index of powerlaw variation of wall temperature n.. We see that the dimensionless velocity and the dimensionless temperature decrease as the parameter $n$ increases

Table (1) represents values of $\mathrm{f}^{\prime \prime}(0)$ and $-\theta^{\prime}(0)$ for various values of $\theta_{\mathrm{r}}$, $\mathrm{M}$ and $\beta$. It is clear that, with increasing $\theta_{\mathrm{r}}, \mathrm{M}$ and $\beta, \mathrm{f}^{\prime \prime}(0)$ and $-\theta^{\prime}(0)$ decrease. It is hoped that the present work will serve as a vehicle for understanding more complex problems involving the various physical effects investigated in the present problem.

\section{Conclusions:}

This work studied the effects of variable viscosity, stretching parameter, the index of power-law variation of wall temperature, the index of power-law velocity and magnetic field on hydromagnetic flow and heat transfer. The governing fundamental equations are transformed into a system of nonlinear ordinary differential equations by similarity transformation and are solved numerically by using shooting method. The numerical results indicate that the variable viscosity parameter increases as the dimensionless velocity decreases but, the temperature distribution increases. Also, that the velocity increases while the temperature decreases and $\mathrm{M}$ increases, further more, that the velocity decreases with increasing the parameter $\beta$, but the temperature increases with increasing $\beta$. The results show that the velocity and the temperature increase as the parameter $\mathrm{M}$ increases, but, with increasing $\theta \mathrm{r}, \mathrm{M}$ and $\beta$, we found $\mathrm{f}^{\prime \prime}(0)$ and ${ }^{-\theta^{\prime}(0)}$ decrease. 


\section{References}

1. B.C. Sakiadis, AIChE J. 7, 221 (1961).

2. LE Erickson, LT Fan and L.C. Cha, AIChE J. 11, 29 (1965)

3. D. Moalem, Int. J. Heat Mass Transfer 19, 529 (1976).

4. I. A. Hassanien, ZAAMZ. Angew Math. Mech. 79, 786 (1999).

5. M. V.Karwe, Y. Jaluria, J. Heat Transfer, 110, 655 (1988).

6. M. A. Seddeek, Int. Comm. Heat Mass Trans. 27, 1037 (2000).

7. M. A. Seddeek, Canadian J. of Physics, 79 (4), 725 (2001).

8. M. A. Seddeek and A. M. Salem, Physics letters A 353, 337 (2006)

9. H. B Pavlov, Magnitnaya Gidrodinamika (USSR) 4, 146 (1974).

10. A. Chakrabarti and A. S. Gupta, Q. Appl. Math 37, 73 (1979).

11. T. C. Chiam, Int. J. Engng. Sci., 33, 429 (1995).

12. J. C. Slattery, "Momentum, Energy and Mass Transfer in Continua", McGraw Hill, New York, (1972).

13. S. P. Anjali Devi and M. Thiyagarajan, Heat Mass Transfer, 42, 671 (2006). 